\title{
Chirurgia laparoscopica e calcolosi renoureterale
}

\author{
R. Sanseverino
}

U.O.C. Urologia, Ospedale Umberto I, Azienda Sanitaria Provinciale di Salerno

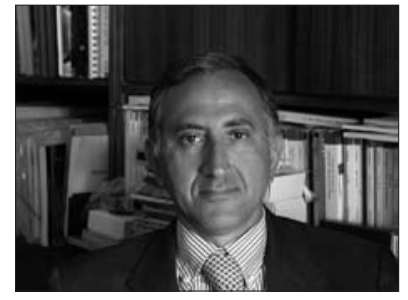

\section{Introduzione}

Il trattamento chirurgico della calcolosi renoureterale è radicalmente cambiato con l'introduzione nella pratica clinica delle metodiche endoscopiche [nefrolitotomia percutanea (PCNL), ureterorenoscopia (URS)] e della litotrissia extracorporea (ESWL). Nella seconda metà degli anni Ottanta c'è stato un forte sbilanciamento in favore della ESWL, che veniva vista come la procedura di gran lunga meno invasiva. Il maturare delle esperienze cliniche in tutto il mondo ed una maggiore consapevolezza riguardo ad un più corretto utilizzo delle nuove metodiche mininvasive, ha portato ad un progressivo riequilibrio delle procedure, a favore in particolare della PCNL, con una costante riduzione al ricorso alle procedure chirurgiche a cielo aperto (1).

In una casistica personale non pubblicata di 741 casi trattati fra il 2002 e il 2005 la distribuzione delle procedure è stata la seguente: ESWL 52\%, URS 29\%, PCNL 18\%, ALTRO 1\% (Fig. 1).

L'introduzione delle procedure laparoscopiche nell'armamentario chirurgico dell'Urologo ha inevitabilmente posto il problema di quali indicazioni potessero trovare le tecniche laparoscopiche nel trattamento moderno della calcolosi renoureterale.

\section{Chirurgia laparoscopica e calcolosi renale}

La diffusione delle procedure laparoscopiche nella comunità urologica internazionale è un fenomeno relativamente recente, tanto che nelle Linee Guida promulgate dalla American Urological Association (AUA) nel 2005 sul trattamento della calcolosi a stampo, il ricorso alle tecniche laparoscopiche non viene consigliato nella routine clinica (2).
Il crescere della esperienza clinica e la comparsa di alcune pubblicazioni scientifiche in materia, hanno reso possibile l'incorporazione delle procedure laparoscopiche nelle opzioni terapeutiche nel trattamento della calcolosi calcica di dimensioni $\leq 20 \mathrm{~mm}$ (superficie $\leq 300$ $\mathrm{mm}^{2}$ ) ed in quella di dimensioni $\geq 20 \mathrm{~mm}$ (superficie $\geq 300 \mathrm{~mm}^{2}$ ), come riportato nelle Linee Guida della European Association of Urology (EAU) del 2008, con livelli di evidenza variabili dal $2^{\circ}$ al $4^{\circ}(3)$.

Lo stesso discorso vale per il trattamento della calcolosi cistinica, particolarmente dura e resistente alle procedure di litotrissia extra o intracorporea (3).

La chirurgia laparoscopica dimostra la propria superiorità nelle procedure demolitive (Fig. 2), in termini di riduzione della degenza e del dolore postoperatorio e di una più precoce ripresa della attività lavorativa [Linee Guida Associazione Urologi Italiani (AURO.it) 2007] (4). L'unica vera controindicazione è rappresentata dalla presenza di tenaci aderenze infiammatorie, che possano rendere difficile e pericolosa la dissezione del peduncolo vascolare. Nella nostra personale esperienza le complicanze più frequenti di questo tipo di chirurgia sono di natura settica e possono portare anche alla formazione di raccolte ascessuali nella loggia di nefrectomia (Fig. 3).

Il ricorso alla laparoscopia può essere giustificato dopo il fallimento di precedenti trattamenti (ESWL, PCNL), come procedura sostitutiva della chirurgia a cielo aperto (4); quanto detto può essere esteso anche alla patologia pediatrica (5).

Un ambito di applicazione specifica delle metodiche laparoscopiche è quello della calcolosi renale in presenza di particolari anomalie renali, come anomalie di fusione (rene a ferro di cavallo), o di posizione (rene pelvico). Nel caso del rene a ferro di cavallo, la ESWL è spesso meno efficace per la maggiore distanza cute via escretrice e la PCNL spesso più complessa e rischiosa per la posizione più mediale e profonda del rene e per la frequente concomitanza di anomalie vascolari. L'acces- 


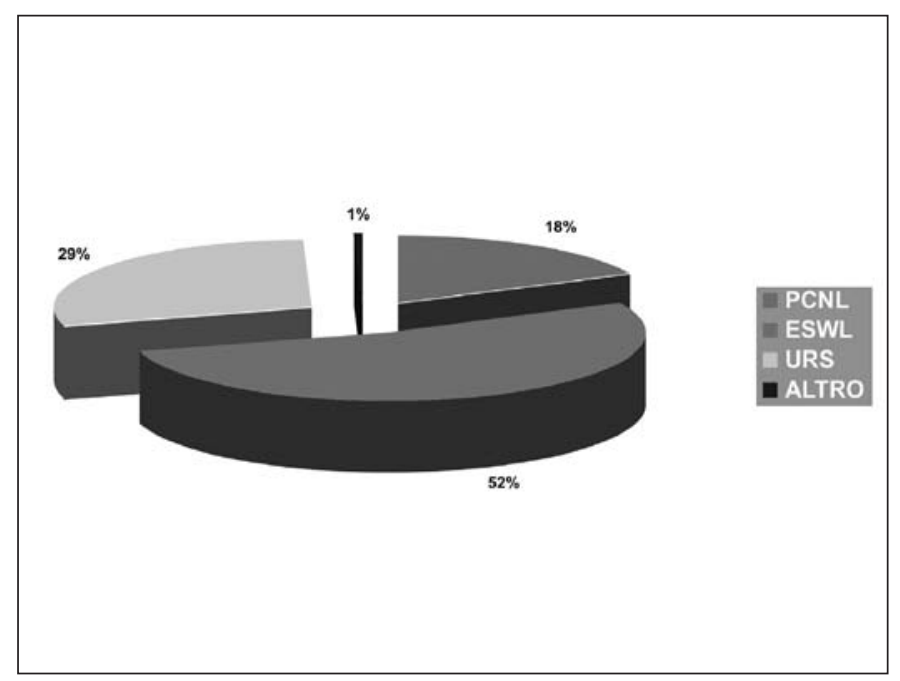

Fig. 1 - Distribuzione delle procedure per calcolosi renoureterale 741 casi 2002-2005.

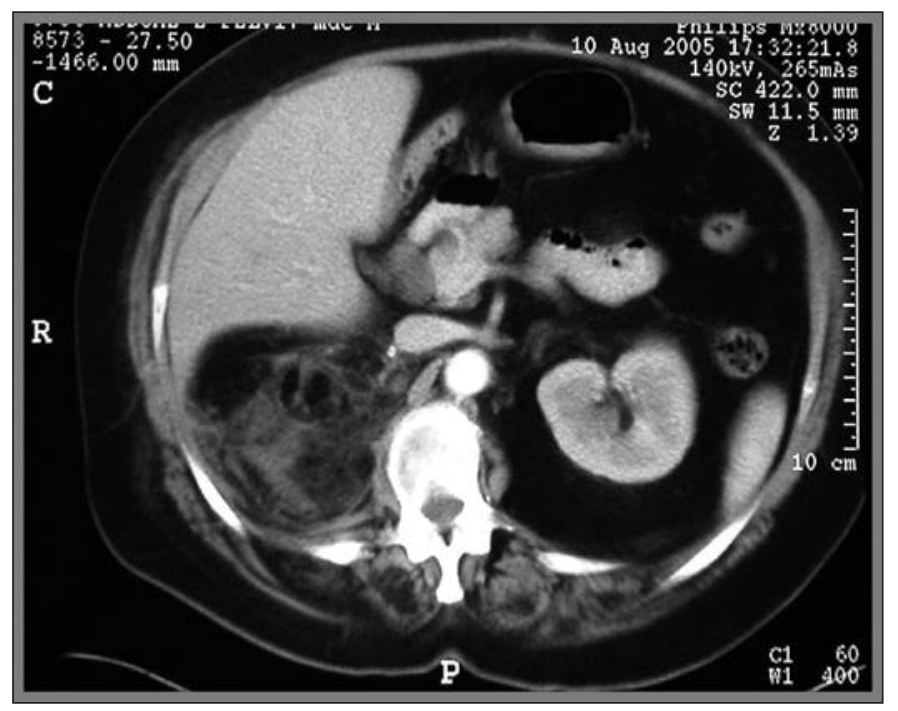

Fig. 3 - Ascesso in loggia renale destra dopo nefrectomia laparoscopica; caso personale.

so laparoscopico avviene abitualmente per via transperitoneale, con approccio diretto alla pelvi renale 0 , più raramente, per realizzare un approccio percutaneo laparoassistito $(6,7)$.

Anche in caso di rene pelvico, la laparoscopia può essere utilizzata come metodica terapeutica primaria o accessoria per un accesso percutaneo laparoassistito (8).

Esistono poi delle condizioni patologiche particolari nelle quali la laparoscopia può trovare uno spazio specifico. L'associazione di stenosi del giunto pieloureterale e di calcolosi renale si presta al trattamento laparoscopico di pielolitotomia e pieloplastica. Il vero problema è rappre-

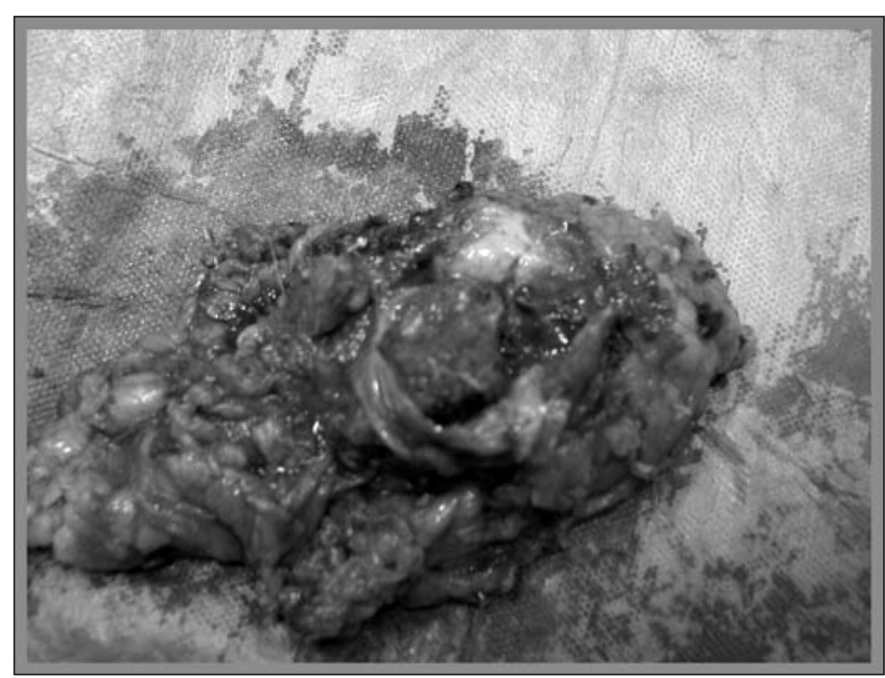

Fig. 2 - Nefrectomia per idropionefrosi calcolosa; caso personale.

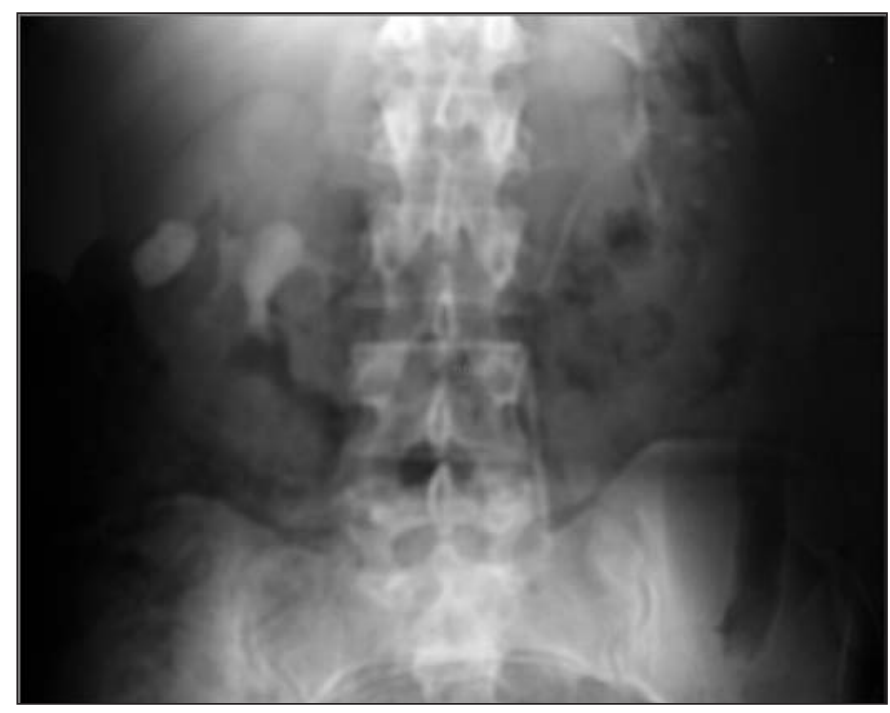

Fig. 4 - Voluminosa calcolosi in diverticolo pielocaliceale.

sentato dalla estrazione dei calcoli renali, naturalmente più agevole in caso di calcolosi singola che di calcolosi multipla. L'estrazione del calcolo può avvenire con l'ausilio di pinze meccaniche, mediante lavaggio a pressione delle cavità renali o mediante contestuale esplorazione endoscopica delle cavità renali. Quest'ultimo procedimento prevede la combinazione di approccio laparoscopico e necroscopia flessibile con visualizzazione diretta ed estrazione dei calcoli; si tratta, nella nostra esperienza di una procedura elegante ed efficace, ma tecnicamente complessa (9).

La presenza di calcolosi in diverticolo pielocaliceale si 
presta al trattamento laparoscopico in caso di calcoli voluminosi, ricoperti da sottile parenchima (Fig. 4) (10).

\section{Chirurgia laparoscopica e calcolosi ureterale}

Le Linee Guida della AUA consigliano il ricorso alla laparoscopia in caso di insuccesso di pregressi trattamenti $o$ in caso di scarsa probabilità che i trattamenti più convenzionali (ESWL, PCNL, URS) possano essere risolutivi (11).

Anche la Linee Guida AURO ribadiscono come la laparoscopia possa essere indicata in caso di calcolosi ureterale di notevoli dimensioni o di precedenti fallimenti (4).

\section{CONCLUSIONI}

La chirurgia laparoscopica può essere utilizzata nel trattamento della calcolosi renoureterale in situazioni particolari, con risultati relativi alla rimozione del calcolo simili a quelli della chirurgia a cielo aperto. Anche le complicanze, a parte quelle proprie della metodica, sono simili (calcolosi residua, sepsi, fistola ruinosa, stenosi ureterale), essendo legate più alla patologia che alla metodica chirurgica utilizzata.

Alcuni ricercatori hanno iniziato ad estendere le indicazioni a situazioni chirurgiche più complesse, con approcci che conservano, per il momento, un carattere del tutto sperimentale (12).

Resta il fatto che la chirurgia laparoscopica rappresenta una metodica di nicchia nel trattamento della calcolosi renoureterale, assestandosi nelle principali casistiche intorno all' $1 \%$ delle procedure.

\section{Indirizzo degli Autori: \\ Roberto Sanseverino, MD \\ Direttore UOC Urologia \\ Ospedale Umberto I \\ Azienda Sanitaria Provinciale di Salerno \\ Salerno, Italy \\ roberto.sanseverino@alice-it}

\section{Bibliografia}

1. Skolarikos A, et al. Percutaneous nephrolithotomy and its legacy. Eur Urol 2005; 47: 22-8.

2. AUA Clinical Guidelines "Staghorne stones", 2005; www. auanet.org

3. EAU Clinical Guidelines "Renal stones", 2008; www.uroweb.org

4. Linee Guida AURO “Calcolosi urinaria”, 2007; www.auro.it

5. Casale P, et al. Transperitoneal laparoscopic pyelolithotomy after failed percutaneous access in the pediatric patient. J Urol 2004; 172(2): 680-3.

6. Ghevariya JD, et al. Laparoscopic pyelolithotmy in horseshoe kidney ñ a case report. J Endourol 2004; 18(Suppl): V23-1.

7. Obek K, et al. Laparoscopic pyelolithotmy in a horseshoe kidney. J Endourol 2004; 18(Suppl): V23-4.

8. Agrawal MS, et al. Laparoscopy-guided PCNL in ectopic (pelvic) kidneys. J Endourol 2004; 18(Suppl): MP27-14.

9. Sanseverino R, et al. Pieloplastica laparoscopica associata a litolapassi mediante nefroscopia flessibile. $3^{\circ}$ Congresso Nazionale Club Litiasi Urinaria: La Terapia Endourologica della Calcolosi. Premio della Giuria come miglior video, Napoli 2-4 aprile 2009.

10. Scott MD, et al. Laparoscopic management of caliceal diverticular calculi. J Urol 2002; 167 (3): 1248-52.

11. AUA Clinical Guidelines "Ureteral stones", 2007; www. auanet.org

12. Deger S, et al. Laparoscopic anatrophic nephrolithtomy. Scand J Urol Nephrol 2004; 38(3): 263-5. 